\title{
ARTICLE The WRKY transcription factor HpWRKY44 regulates CytP450-likel expression in red pitaya fruit (Hylocereus polyrhizus)
}

\author{
Mei-nv Cheng, Zi-juan Huang, Qing-zhu Hua, Wei Shan, Jian-fei Kuang, Wang-jin Lu, Yong-hua Qin and Jian-ye Chen
}

Red pitaya (Hylocereus polyrhizus) fruit is a high-value, functional food, containing a high level of betalains. Several genes potentially related to betalain biosynthesis, such as cytochrome P450-like (CytP450-like), have been identified in pitaya fruit, while their transcriptional regulation remains unclear. In this work, the potential involvement of a WRKY transcription factor, HpWRKY44, in regulating CytP450-like 1 expression in pitaya fruit was examined. HpWRKY44, a member of the Group 1 WRKY family, contains two conserved WRKY motifs and is localized in the nucleus. HpWRKY44 also exhibits trans-activation ability. Gene expression analysis showed that the expression of HpCytP450-like1 and HpWRKY44 increased steadily during pitaya fruit coloration, which corresponded with the production of elevated betalain levels in the fruit. HpWRKY44 was also demonstrated to directly bind to and activate the HpCytP450-like1 promoter via the recognition of the W-box element present in the promoter. Collectively, our findings indicate that HpWRKY44 transcriptionally activates HpCytP450-like1, which perhaps, at least in part, contributes to betalain biosynthesis in pitaya fruit. The information provided in the current study provides novel insights into the regulatory network associated with betalain biosynthesis during pitaya fruit coloration.

Horticulture Research (2017) 4, 17039; doi:10.1038/hortres.2017.39; Published online 2 August 2017

\section{INTRODUCTION}

Betalains are naturally occurring, water-soluble pigments produced from amino acid L-tyrosine. More than 50 natural betalains have been reported and they are present only in species within the order Caryophyllales, such as beets, Amaranthus, prickly pear cactus and red pitaya fruit. ${ }^{1-3}$ The red-violet betacyanins and the yellow betaxanthins, are the two types of betalains. ${ }^{4}$ These pigments are not produced in the same plant or at the same time when anthocyanins, another major pigment group, are present. ${ }^{5}$ Betalains are widely used as economically important natural food colorants and functional foods. ${ }^{6,7}$ Therefore, understanding the regulatory mechanisms responsible for betalain biosynthesis could provide information to assist the development of biotechnological approaches aimed to improve betalain production in plants.

The biochemical pathway of betalain biosynthesis from tyrosine has been extensively studied. ${ }^{1,8-10}$ Enzymes involved in betalain biosynthesis, such as tyrosinase (TYR), 4,5-dihydroxy-phenylalanine (DOPA)-dioxygenase (DOD) and glucosyltransferases (GTs) have been identified in higher plants. ${ }^{1,8-10}$ It has been proposed that TYR converts tyrosine to L-3,4-dihydroxyphenylalanine (L-DOPA), which in turn is converted to betalamic acid by DOD. GTs, the last family of enzymes in the pathway, make betalains stable and diversify their composition by transferring sugar residues. $^{1,8-10}$ Three cytochrome P450-type enzymes, CYP76AD1, CYP76AD5 and CYP76AD6, have been well documented to be associated with the hydroxylation of tyrosine to form L-DOPA. In addition, CYP76AD1 has also been shown to function exclusively in the formation of 5,6-dihydroxyindoline-2-carboxylic acid (cyclo-
DOPA) from L-DOPA in red beet. ${ }^{9,11-13}$ Genes encoding these enzymes have been identified in plant species within the Caryophyllales and their expression levels have been demonstrated to increase with betalain accumulation, suggesting that betalain biosynthesis is regulated by the transcription level of the genes encoding these enzymes. ${ }^{1}$ Recently, a betalain biosynthetic gene, CYP76AD1 has been demonstrated to be transcriptional regulated by a MYB-family transcription factor (TF). ${ }^{14}$ This is the first TF reported to be involved in the regulation of the betalain biosynthesis. Although thousands of TFs have been identified in plants, however, the identification and role of other TFs regulating betalain biosynthesis remains largely unknown.

As a large family of plant-specific TFs, the first WRKY protein is identified in sweet potato. ${ }^{15}$ Numerous WRKY genes have now been isolated and characterized in many plants. For instance, $>197,100,74,287$ and 62 WRKY members have been identified in Glycine max, Oryza sativa, Arabidopsis, Brassica napus and Fragaria vesca, respectively. ${ }^{16-20}$ Two highly conserved domains are found in WRKY proteins, one is WRKY with WRKYGQK sequences, and the other is a C-terminal zinc-finger motif of Cys and His residues. ${ }^{16,21}$ Plant WRKY proteins are well-known to be involved in various stress responses, hormone signaling, morphogenesis of trichomes and embryos, and senescence. ${ }^{20,22-24}$ WRKYs are also reported to regulate plant metabolite biosynthesis, including phenylpropanoids, alkaloids and terpenes, by regulating metabolite biosynthetic genes. ${ }^{25-28}$ Betalains are also secondary metabolites, however, the involvement of WRKY TFs in betalain biosynthesis, especially in economical fruits, has been largely unexplored.

State Key Laboratory for Conservation and Utilization of Subtropical Agro-bioresources/Guangdong Provincial Key Laboratory of Postharvest Science of Fruits and Vegetables/Key

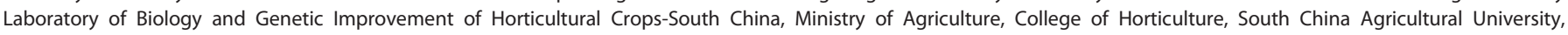
Guangzhou 510642, China.

Correspondence: Y-h Qin (qinyh@scau.edu.cn) and J-y Chen (chenjianye@scau.edu)

Received: 5 April 2017; Revised: 19 June 2017; Accepted: 26 June 2017 
Pitayas, also called as dragon fruit, belongs to the genus Hylocereus in the order Caryophyllales, and is commercially cultivated due to its nutritional value. ${ }^{29}$ There are several species of pitayas, while the most widely cultivated are Hylocereus undatus and Hylocereus polyrhizus, which have white and red colored pulp, respectively. ${ }^{30}$ As red pulp pitaya contains high levels of betalains, most of the previous studies of this fruit have focused on purification and characterization of betalins. ${ }^{30,31}$ Recently, several putative genes, including TYR, DOD-like, CytP450-like and GT-like, were identified in red pulp pitaya using RNA-seq, and associated with betalain biosynthesis. ${ }^{10}$ In the present work, the possible association of a Group I WRKY TF, HpWRKY44, with the direct activation of HpCytP450-like1 is reported, providing new information on the transcriptional control of betalain production in pitaya fruit.

\section{MATERIALS AND METHODS}

\section{Fruit samples}

Red pitaya plants (Hylocereus polyrhizus cv. Dayeshuijing) were grown under field conditions at a local commercial plantation in Guangzhou, China. Fruits were sampled on the $16^{\text {th }}, 21^{\text {tt }}, 26^{\text {th }}, 30^{\text {th }}, 35^{\text {th }}, 40^{\text {th }}$ and $49^{\text {th }}$ day after artificial pollination (DAAP). The sample dates were selected to provide a set of samples covering pulp color changes in the fruit. At each sampling, internal fruit tissue (pulp tissue) from three fruits from three different plants were sampled, sliced, and frozen in liquid nitrogen immediately. Samples were stored at $-80^{\circ} \mathrm{C}$ for future analysis.

\section{Betalain quantification}

The level of betalains in the pulp tissue of each sample was determined as previously described. ${ }^{10}$ Betalains were isolated from $0.5 \mathrm{~g}$ pulp with $5 \mathrm{~mL}$ $80 \%$ aqueous methanol $(\mathrm{v} / \mathrm{v})$ solution. The amount of betacyanin and betaxanthin in the solutions were measured by spectrophotometry (Infinite M200, Tecan Co.) at 538 and $483 \mathrm{~nm}$, respectively, and reported as $\mathrm{mg} / 100 \mathrm{~g}$ fresh pulp.

\section{Gene isolation and sequence analysis}

Total RNA was extracted from pitaya pulp samples using the Quick RNA Isolation Kit (Huayueyang, Beijing, China) following manufacturer's instructions. RNA quality was assessed by gel electrophoresis and spectrophotometry. The extracted RNA was used as template to synthesis CDNAs using a PrimeScript RT reagent Kit with gDNA Eraser (TaKaRa, shiga, Japan).

On the basis of the generation of a previous RNA-seq database, ${ }^{10}$ a WRKY TF was found to be significantly up-regulated during pulp coloration. As this WRKY displays high degree of sequence homology to Arabidopsis thaliana WRKY44, thus it was termed as HpWRKY44. HpWRKY44 was cloned (primers are listed in Supplementary Table S1), sequenced and the resulting sequence was queried against the NCBI database in order to identify homologous genes. The theoretical isoelectric point (pl) and mass value for HpWRKY44 protein were determined following the method described at http://web.expasy.org/compute_pi/. Sequences were aligned using CLUSTALW (version 1.83) and GeneDoc software. A phylogenetic tree was constructed using the Neighbor-Joining method in MEGA5.0.

\section{Gene expression analysis}

Gene expression was analyzed by reverse transcription-quantitative PCR (RT-qPCR) using GoTaq qPCR Master Mix Kit (Promega, Madison, WI, USA) as described previously. ${ }^{10}$ The PCR was conducted on a Bio-Rad CFX96 Real-Time PCR System. The cycling began with an initial denaturation step at $94^{\circ} \mathrm{C}$ for $5 \mathrm{~min}$, followed by 40 cycles of $94^{\circ} \mathrm{C}$ for $10 \mathrm{~s}, 60^{\circ} \mathrm{C}$ for $30 \mathrm{~s}$, and $72^{\circ} \mathrm{C}$ for $30 \mathrm{~s}$. A no-template control and melting curve analysis was included in every PCR run. Relative gene expression was normalized according to the cycle threshold $(\mathrm{Ct})$ value using actin as the reference gene. Primers used in the RT-qPCR analysis are listed in Supplementary Table S1.

\section{Subcellular localization of HpWRKY44}

To determine the cellular localization of HpWRKY44, its full-length was cloned into $\mathrm{PEAQ}-\mathrm{GFP}$ for fusion with a GFP reporter gene (primers are listed in Supplementary Table S1). The Agrobacterium tumefaciens strain
GV3101 cells carrying pEAQ-HpWRKY44-GFP or the GFP positive control were infiltrated into tobacco (Nicotiana benthamiana) leaves as previously described. $^{32,33}$ Transient expression of GFP was recorded using a fluorescence microscope (Zeiss Axioskop 2 Plus) at $500 \mathrm{~nm}$ after two days of infiltration.

\section{HpCytP450-like1 promoter analysis}

A CTAB-based method was used to extract genomic DNA from pitaya pulps. ${ }^{34}$ The HpCytP450-like1 promoter was isolated with a Genome Walker Kit (Clontech) using a nested PCR approach. The sequence of HpCytP450like1 promoter was subjected to the Plant-CARE database (http:// bioinformatics.psb.ugent.be/webtools/plantcare/html/) for the prediction of conserved cis-element motifs. Specific primers are listed in Supplementary Table S1.

\section{Electrophoretic mobility shift assay}

The N-terminus of HpWRKY44, including the WRKY domain (from 574 to 1194 bp), was inserted into pGEX-4T-1 (GE Healthcare Life Sciences (China), Beijing, China) to construct a GST-HpWRKY44 expression vector, and introduced into Escherichia coli strain BM Rosetta (DE3). GST-HpWRKY44 fusion protein was purified using Glutathione-Superflow Resin (Clontech, California, United States) after induction by $1 \mathrm{~mm}$ isopropyl thio- $\beta-D-$ galactoside (IPTG) at $30{ }^{\circ} \mathrm{C}$ for $6 \mathrm{~h}$.

LightShift Chemiluminescent EMSA Kit (Thermo Scientific, Illinois, United States) was used to perform EMSA as previously described. ${ }^{33,35}$ DNA fragments, including an oligonucleotide harboring two W-box (TTGAC) motifs and the HpCytP450-like1 promoter with $74 \mathrm{bp}$ containing two consensus W-box motifs were biotin-labeled at the $5^{\prime}$ end (primers are listed in Supplementary Table S1). The same or mutated probes without biotin labeling were used as the competitors. These probes were incubated with purified GST-HpWRKY44 fusion protein, and the GST protein alone was used as the negative control. After SDS-PAGE separation, protein-DNA complexes were detected using the chemiluminescence method and photos were taken on a ChemiDoc MP Imaging System (Bio-Rad, Hercules, CA, USA).

\section{Transcriptional activity}

Dual-luciferase transient expression system in tobacco leaves was adopted to investigate the transcriptional activity of HpWRKY44. The reporter vector was constructed based on the pGreenll 0800-LUC vector. ${ }^{36}$ The minimal TATA box of the CaMV 35S promoter plus five copies of the GAL4 DNAbinding element $(5 \times G A L 4)$ were placed in front of the firefly luciferase (LUC). CaMV 35S-driving Renilla luciferase (REN) in the same vector was considered as an internal control. For the effector vector, the full-length of HpWRKY44 fusing with the yeast GAL4 DNA-binding domain (GAL4BD) was droven by CaMV $35 \mathrm{~S}$.

In order to assess the specific binding and activity of HpWRKY44 to

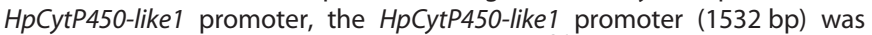
inserted into the pGreenll0800-LUC vector, ${ }^{36}$ while HpWRKY44 was inserted into the $\mathrm{pEAQ}$ vector as an effector. Primers for all constructs are listed in Supplementary Table S1.

A total of $2.5 \mu \mathrm{g}$ plasmid DNA of the reporter and the effector were co-transformed into tobacco leaves in each assay, as described above. Two days after inoculation, LUC and REN luciferase activity was quantified as described previously. ${ }^{33}$ The transcriptional activity of HpWRKY44, as well as the trans-activation of HpCytP450-like1 by the HpWRKY44, were calculated as the ratio of LUC/REN. For each pair, at least six independent replicates were assessed.

\section{Data analysis}

At least three individual biological replicates were utilized in all of the conducted analyses. Data represent the mean \pm s.e.m. A one-way ANOVA was performed to determine the significance of experimental means at $P<0.05$ and $P<0.01$.

\section{RESULTS AND DISCUSSION}

Changes in betacyanin and betaxanthin content during color development of pitaya fruit pulp color

In pitaya fruit there are two types of betalains which are reddish violet (betacyanins) and yellow (betaxanthins). ${ }^{1}$ As illustrated in 


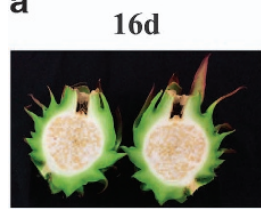

$35 d$

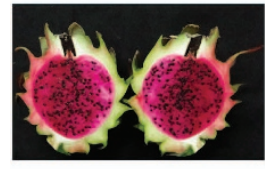

21d

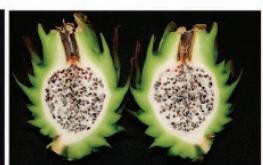

40d

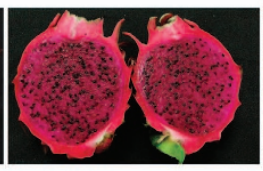

26d

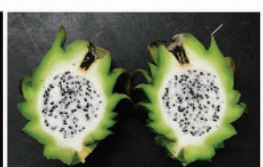

49d

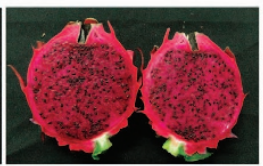

30d

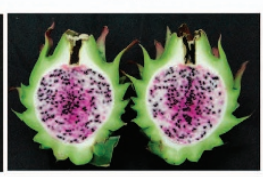

\section{b}

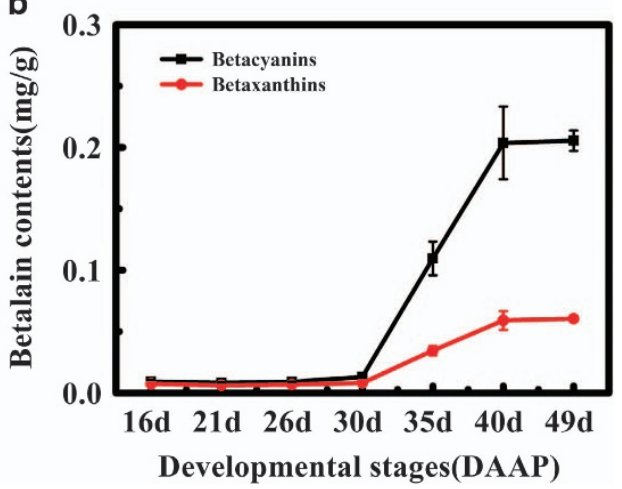

Figure 1. Pitaya fruit pulp at different developmental stages (a) and changes in betalain content (b) during fruit pulp color formation. Fruits were sampled for subsequent analyses at $16^{\text {th }}, 21^{\text {st }}, 26^{\text {th }}, 30^{\text {th }}, 35^{\text {th }}, 40^{\text {th }}$ and $49^{\text {th }}$ days after artificial pollination (DAAP). Data represent mean \pm s.e.m. of three biological replicates $(n=3)$.

\begin{tabular}{|c|c|c|c|}
\hline ABRE & $719(-) ; 107(-)$ & $\begin{array}{l}\text { TACGTG } \\
\text { CGTACGTGCA }\end{array}$ & $\begin{array}{l}\text { cis-acting element involved in the abscisic acid } \\
\text { responsiveness }\end{array}$ \\
\hline ACE & $852(+)$ & AAAACGTTTT & cis-acting element involved in light responsiveness \\
\hline ARE & $225(+)$ & TGGTTT & $\begin{array}{l}\text { cis-acting regulatory element essential for the anaerobic } \\
\text { induction }\end{array}$ \\
\hline ATGCAAAT motif & $688(-)$ & ATACAAAT & $\begin{array}{l}\text { cis-acting regulatory element associated to the TGAGTCA } \\
\text { motif }\end{array}$ \\
\hline CAAT-box & $244(-) ; 703(-) ; 1256(-)$ & $\begin{array}{l}\text { CCAAT } \\
\text { GGCAAT }\end{array}$ & $\begin{array}{l}\text { Common cis-acting element in promoter and enhancer } \\
\text { regions }\end{array}$ \\
\hline CAT-box & $1131(+) ; 1454(+)$ & GCCACT & $\begin{array}{l}\text { cis-acting regulatory element related to meristem } \\
\text { expression }\end{array}$ \\
\hline CATT-motif & $45(+) ; 70(+)$ & GCATTC & Part of a light responsive element \\
\hline CCAAT-box & $1086(+)$ & CAACGG & MYBHv1 binding site \\
\hline GARE-motif & $292(+)$ & TCTGTTG & Gibberellin-responsive element \\
\hline GATA-motif & $849(-)$ & AAGGATAAAG & Part of a light responsive element \\
\hline Gap-box & $1356(-)$ & CAAATGAA(A/G)A & Part of a light responsive element \\
\hline HSE & $1485(-)$ & AAAAAATATC & cis-acting element involved in heat stress responsiveness \\
\hline LAMP-element & $1418(+)$ & CTTTATCA & Part of a light responsive element \\
\hline MBS & $318(+)$ & TAACTG & MYB binding site involved in drought-inducibility \\
\hline MRE & $340(+) ; 345(+)$ & AACCTAA & MYB binding site involved in light responsiveness \\
\hline Skn-1_motif & $1480(+) ; 546(-) ; 959(+) ; 896(-) ; 983(-)$ & GTCAT & $\begin{array}{l}\text { cis-acting regulatory element required for endosperm } \\
\text { expression }\end{array}$ \\
\hline TATA-box & $\begin{array}{c}952(-) ; 156(-) ; 332(-) ; 573(-) ; 619(-) ; 1034 \\
(+) ; 747(-) ; 635(+)\end{array}$ & $\begin{array}{l}\text { TATTTAAAA } \\
\text { TATA } \\
\text { TATAAA } \\
\text { TATAA }\end{array}$ & Core promoter element around -30 of transcription start \\
\hline TGACG-motif & $960(-)$ & TGACG & $\begin{array}{l}\text { cis-acting regulatory element involved in the } \\
\text { MeJA-responsiveness }\end{array}$ \\
\hline
\end{tabular}

Figures $1 \mathrm{a}$ and $\mathrm{b}$, both betacyanin and betaxanthin content began to increase with the onset of color development in the pulp beginning at 30 DAAP. Levels steadily increased afterwards and the whole pulp was deep red by 49 DAAP.

Promoter analysis of HpCytP450-like1

Previous transcriptomic and RT-qPCR analysis revealed that among the identified putative genes that are potentially related to betalain biosynthesis, the expression level of two CytP450 genes (HpCytP450-like1 and HpCytP450-like4), and one DOD gene (DODAlike2), increased gradually during the development of the red pulp color, and that the gradual increase in the expression of these genes corresponded with the accumulation of betalain during the transition of pulp color from white to red. ${ }^{10} \mathrm{~A}$ database query indicated that the amino acid sequence of HpCytP450-like 1 and DODA-like2 shared high identity with BvCYP76AD1 (74\%) and 

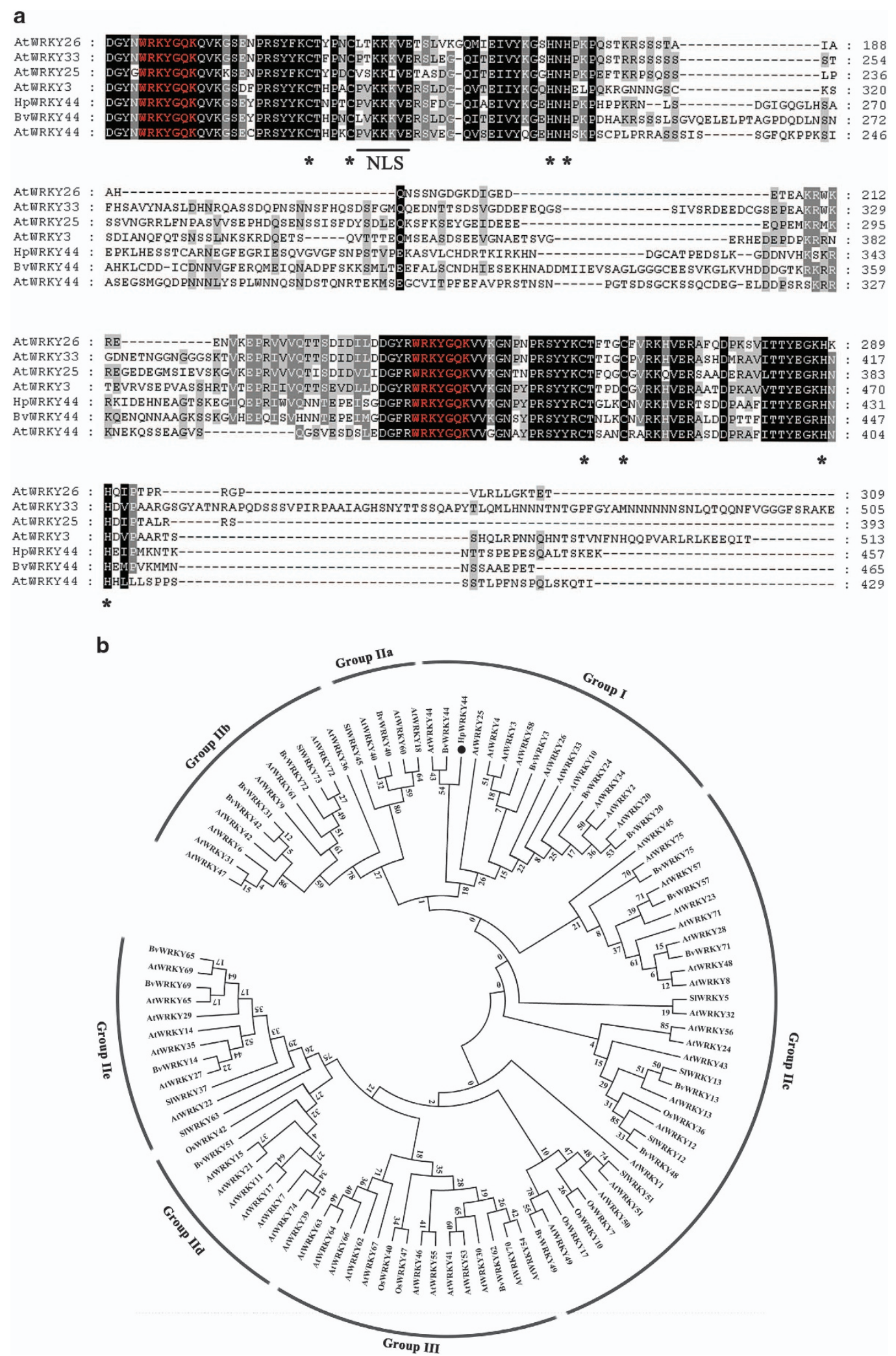

Figure 2. Bioinformatic analysis of HpWRKY44. (a) Multiple alignment of HpWRKY44 with sugar beet BvWRKY44, and Arabidopsis thaliana AtWRKY3, AtWRKY25, AtWRKY26, AtWRKY33 and AtWRKY44. Identical and similar amino acids are represented by black and gray shading, respectively. The two WRKY motifs and the zinc-finger structures are indicated by red letters and asterisks, respectively. A nuclear localization signal (NLS) is underlined. (b) Phylogenetic tree of HpWRKY44, sugar beet, Arabidopsis thaliana, rice and tomato WRKYs. WRKYs are divided into three major groups and seven sub-families. HpWRKY44 (black circles), along with sugar beet, BvWRKY44, Arabidopsis thaliana AtWRKY25, AtWRKY44 and tomato SIWRKY5 cluster in Group I. The phylogenetic tree was constructed with MEGA5.0 using a bootstrap test of phylogeny with UPGMA test and default parameters. 
BvDODA1 (63\%), respectively; two genes that are known to be involved in betalains biosynthesis in red sugar beet.9,14 These homologies indicated that HpCytP450-like1 and DODA-like2 might be related to betalains biosynthesis in pitaya fruit. Further biochemical and molecular evidence are required, however, to support their direct involvement in betalain biosynthesis, and to identify where they belong in the betalain biosynthetic pathway. In the current study, an attempt was made to isolate the promoters of all three of these genes, however, only the cloning of the HpCytP450-like1 promoter was successful. A motif scan of the 1532 bp-long HpCytP450-like1 promoter was conducted using Plant-CARE database in order to identify cis-acting elements. In addition to the core cis-acting elements, such as a TATA box and CAAT box, three typical W-box motifs with a core sequence $(C / T)$ $\mathrm{TGAC}(\mathrm{C} / \mathrm{T})$ (Supplementary Text S1) were identified in the HpCytP450-like1 promoter. The W-box is a well-known binding element of WRKY TFs, suggesting the possible involvement of WRKY TFs in regulating HpCytP450-like1. Binding sites for MYB, HSF and AREB/ABF TFs were also identified (Table 1).

\section{Cloning and sequence analysis of HpWRKY44}

WRKY TFs in plants have been implicated in the regulation of phenylpropanoids, alkaloids and terpenes, through their ability to regulate metabolite biosynthetic genes. ${ }^{25-28}$ Betalains are sec-

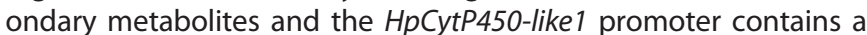
motif suitable for the binding of WRKY TFs (Table 1). This finding prompted further analysis of the relationship between WRKY TFs and betalain biosynthesis genes in pitayas. Our previous RNA-seq study identified a full-length WRKY gene that was up-regulated during color development in pitaya fruit pulp. The full-length sequence of this WRKY gene exhibited high similarity to AtWRKY44 (40\%), and so was named HpWRKY44. The Open Reading Frame of HpWRKY44 is $1371 \mathrm{bp}$ in length, and encodes a polypeptide of 457 amino acids, with a calculated molecular weight of $50.89 \mathrm{kDa}$, and a pl of 9.02. HpWRKY44 has two highly conserved amino acid sequences of WRKYGQK, which is a WRKY domain and a defining characteristic of WRKY TFs. ${ }^{21}$ HpWRKY44 also contains two putative zinc-finger motifs (C-X4-CX23-24-H-X1H) (Figure 2a).

WRKY proteins are clustered into three major groups (I-III), and group II can be further subdivided into five subgroups (Ila-e). ${ }^{16,21}$ A phylogenetic tree was constructed using the amino acid sequence of HpWRKY44, sugar beet, Arabidopsis thaliana, tomato, and rice WRKYs. HpWRKY44 was clustered with Group I WRKYs, along with the BvWRKY44 from sugar beet, and the AtWRKY44, AtWRKY25 and AtWRKY33 from Arabidopsis thaliana (Figure 2b). Notably, AtWRKY33 and AtWRKY44 have been reported to act as transcriptional regulators of phenylpropanoid and indole alkaloid biosynthesis, ${ }^{37,38}$ further indicating a potential role for HpWRKY44 in the biosynthesis of secondary metabolites in pitaya.

Expression of HpCytP450-like1 and HpWRKY44 during color development in pitaya fruit pulp

The expression level of HpCytP450-like 1 and HpWRKY44 during the coloration of the pulp was determined by RT-qPCR in order to examine their relationship to betalain biosynthesis during fruit development. Results indicated that the transcript levels of HpCytP450-like1 and HpWRKY44 were relatively low from 16 to 21 DAAP (Figure 3). Consistent with the accumulation of betalains at 30 DAAP, however, both HpCytP450-like1 and HpWRKY44 transcript levels increased by approximately 910- and 4.1-fold of the initial level at 30 DAAP, respectively. This elevation in expression was coincident with the increase in the red color development of the pulp (Figure 3).

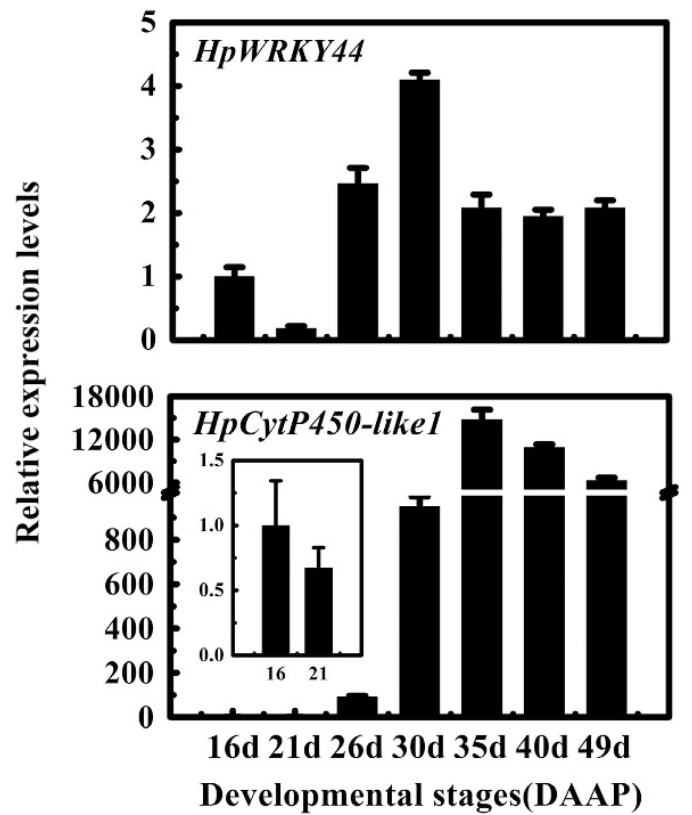

Figure 3. Expression pattern of HpCytP450-like1 and HpWRKY44 during fruit pulp coloration. Fruits were sampled at $16^{\text {th }}, 21^{\text {st }}, 26^{\text {th }}$, $30^{\text {th }}, 35^{\text {th }}, 40^{\text {th }}$ and $49^{\text {th }}$ days after artificial pollination (DAAP). The expression level of each gene is expressed as a ratio relative to 16 DAAP, which was set at 1. Each value represents the mean \pm s.e.m. of three replicates $(n=3)$.

Nuclear localization of HpWRKY44 and trans-activation ability WRKYs, like other TFs, are typically nuclear-localized proteins that possess transcriptional activity. ${ }^{19,20,39,40}$ Sub-cellular localization of HpWRKY44 was determined in tobacco leaves using a GFP-tagged HpWRKY44 protein driven by the CaMV 35 S promoter. As shown in Figure 4, the HpWRKY44-GFP fusion protein was localized to the nucleus, while for the positive control, its GFP signal was observed around the cytoplasm and the nucleus.

The transcriptional activity of HpWRKY44 in plant cells was also analyzed using a dual-luciferase reporter system (Figure 5a). Results of the analysis indicated that compared with the negative control pBD, HpWRKY44 obviously increased the value of the LUC/ REN ratio (Figure 5b). These results indicate that HpWRKY44 is a nuclear protein and may potentially act as a transcriptional activator.

Interaction of HpWRKY44 with the W-box in HpCytP450-like1 promoter in vitro

Previous studies have reported the direct binding of WRKY TFs to the W-box motif within target gene promoters. ${ }^{16,19,20,41}$ For example, the WRKY TF, AaGSW1, of Artemisia annua can directly bind to W-box motifs in the CYP71AV1 promoter. $^{42}$ In the current study, the interaction between HpWRKY44 and the W-box of HpCytP450-like1 promoter was characterized by EMSA. Firstly, glutathione S-transferase (GST)-HpWRKY44 fusion protein was prokaryotic induced and purified successfully (Figure 6a). Results indicated the formation of DNA-protein complexes with reduced migration when the recombinant GST-HpWRKY44 protein was mixed with the biotin-labeled DNA probe with two W-box motifs or with the HpCytP450-like1 promoter containing a single W-box motif (Figure 6b). Formation of the DNA-protein complexes was effectively abolished when the corresponding unlabeled probes were added to the mixture as a cold competitor. but was not abolished when mutated $\mathrm{W}$-box probes were used in the assay (Figure $6 \mathrm{~b}$ ). As expected, the mobility shift was also absent when 
the empty GST protein were incubated with biotin-labeled probes (Figure 6b). Collectively, these results indicate that HpWRKY44 is capable of binding to the $\mathrm{W}$-box motif present in the promoter of HpCytP450-like1.

HpWRKY44 activates the transcription of HpCytP450-like1 in vivo The ability of $H p C y t P 450$-like 1 to activate the transcription of HpWRKY44 was determined using transient dual-luciferase assays in tobacco leaves (Figure 7a). HpWRKY44 was inserted into the $\mathrm{pEAQ}$ vector to serve as an effector, and the empty $\mathrm{PEAQ}$ was

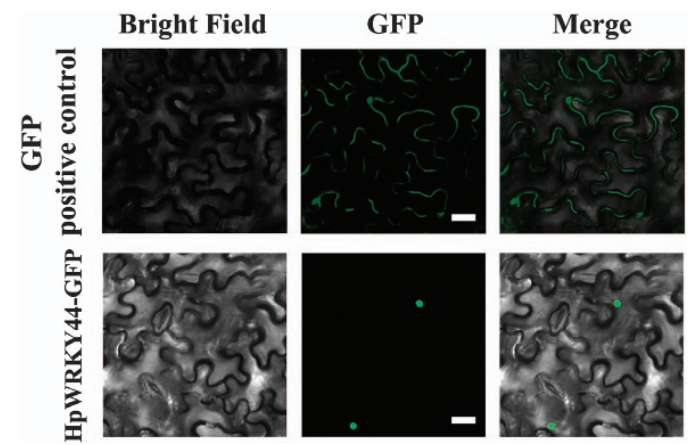

Figure 4. Subcellular localization of HpWRKY44 in tobacco leaves. Agrobacterium tumefaciens carrying HpWRKY44-GFP or a GFP positive control vector were infiltrated into tobacco leaves. After $48 \mathrm{~h}$, the fluorescence of HpWRKY44-GFP protein was localized exclusively in the nucleus, while the fluorescence of the GFP positive control was distributed in both the nucleus and cytoplasm. Bar $=30 \mu \mathrm{m}$. included as a control (Figure 7a). As shown in Figure 7b, overexpression of HpWRKY44 significantly increased the LUC/

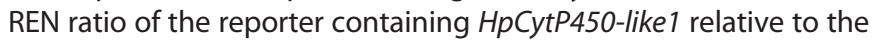
corresponding empty control. These results indicate that HpWRKY44 activated the transcription of HpCytP450-like1 and supports the premise that HPWRKY44 has a functional role in the

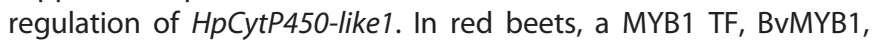
has been reported to be involved in betalain biosynthesis, as silencing of BvMYB1 results in the down-regulation of betalain biosynthetic genes and pigmentation. ${ }^{14}$ BvMYB1 can also target the CYP76AD1 promoter. $^{14}$ Interestingly, MYB and AREB/ABF TFs binding sites are also present in the promoter of HpCytP450-like1. Therefore, whether or not HpCytP450-like1 is also targeted by MYB and AREB/ABF TFs needs to be examined. The identification of additional TFs involved in the regulation of betalain biosynthesis genes in pitaya fruit also need to be identified. Moreover, it has been well documented that regulatory proteins, such as TFs, seldom act alone. Numerous studies have demonstrated that WRKY TFs physically interact with a wide range of proteins that have functional roles in signaling, transcription and chromatin remodeling. These protein interactions can affect the DNA-binding and transcription-regulatory activity that WRKY TFs have with their targets genes. ${ }^{43}$ For example, MaWRKY1 and MaWRKY2 in banana fruit cooperate with a NAC TF, MaNAC5, to activate the expressions of a specific set of $P R$ genes associated with disease response. $^{44}$ In addition, MaVQ5 in banana fruit antagonizes MaWRKY2 6 in the activation of JA biosynthesis in response to cold stress. ${ }^{40}$ Therefore, in future studies, it would be interesting to determine whether or not HpWRKY44 can coordinate with MYB AREB/ABF TFs, or other proteins to regulate betalain biosynthesis.

a

Reporter
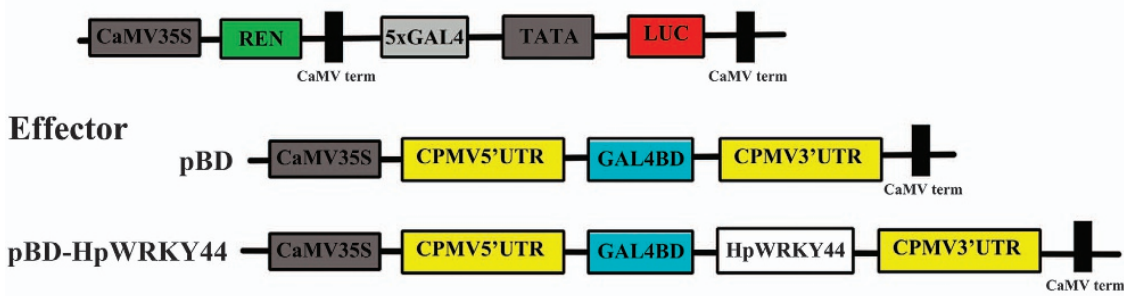

b

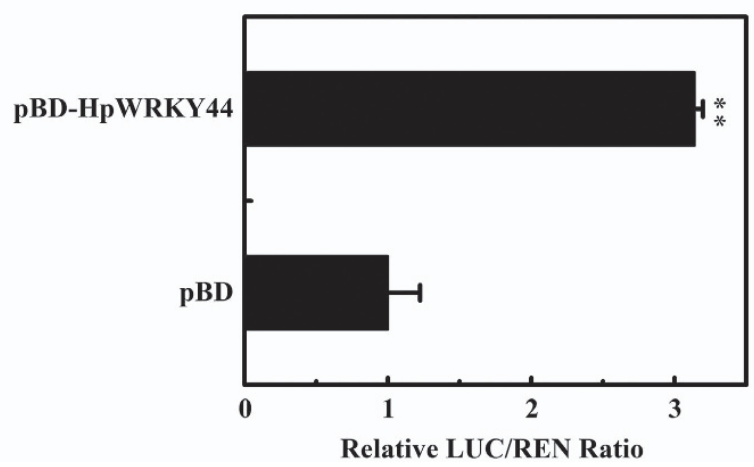

Figure 5. Analysis of transcriptional activity of HpWRKY44. (a) Reporter and effector constructs. The dual luciferase reporter construct contained the firefly luciferase (LUC) reporter gene fused with five copies of the GAL4 DNA-binding element (5 $\times$ GAL4) plus the mini-35S (TATA box). The Renilla luciferase (REN) driven by CaMV 35S in the same vector was used as an internal control. The effector plasmid contained the HpWRKY44 gene fused to the yeast GAL4 DNA-binding domain (GAL4BD) driven by CaMV35S, CPMV, (Cowpea mosaic virus). (b) Transcriptional activation activity of HpWRKY44. The trans-activation ability of HpWRKY44 was assessed as the ratio of LUC to REN. Each presented value represents the mean \pm s.e.m. six biological replicates $(n=6)$. The ratio of LUC/REN of the empty pBD vector was used as calibrator and set at $1 .{ }^{* *}$ indicates a significant difference between the sample (transcriptional activator vector) and the control (empty pBD vector) at $P<0.01$, based on the Student's $t$-test. 
a

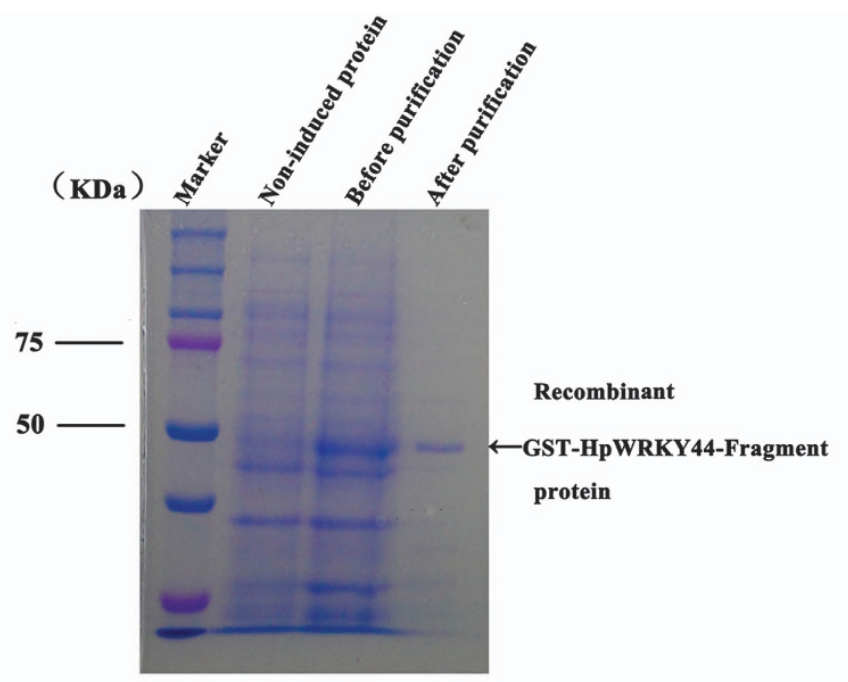

b

W-box

wild: GGAACTTGACCATCCGTTGACCGGGCTGCAGGAATTCG

mutant: GgAACAAAAACATCCGAAAAACGGGCTGCAGGAATTCG

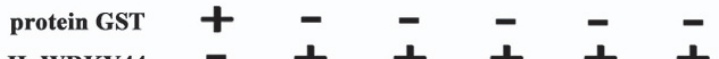

protein GST-HpWRKY44 $-++t+$

Biotin probe $++t+t$

Cold probe - -+++-

Mutant probe $\quad-\quad-\quad-\quad-\quad+++$

shift

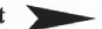

free probe

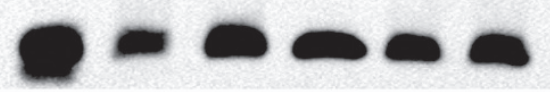

\section{HpCytP450-like1}

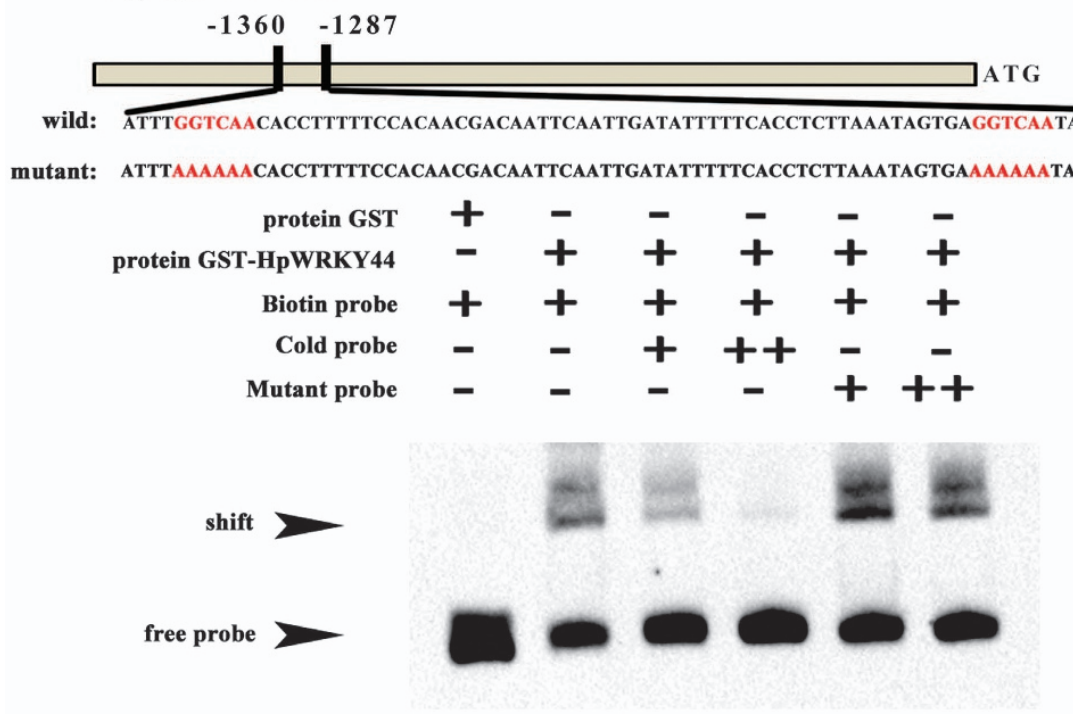

\section{CONCLUSIONS}

In summary, a Group I WRKY TF, HpWRKY44, was cloned and characterized from pitaya fruit. HpWRKY44 activated HpCytP450-like1 expression by binding to its promoter.
To the best of our knowledge, this is the first report of the involvement of WRKY TFs in regulating HpCytP450-like1 in fruits. Overall, our findings provides new insights into the transcriptional regulation of genes associated with betalain 
Figure 6. Electrophoretic mobility shift assay (EMSA) demonstrating the in vitro binding of HpWRKY44 to a W-box element and the HpCytP450like1 promoter containing a W-box element. (a) SDS-PAGE gel stained with Coomassie blue demonstrating affinity purification of the recombinant HpWRKY44 protein used for the EMSA. (b) EMSA. Biotin-labeled DNA probe from the promoter or mutant probe was incubated with GST-HpWRKY44 protein, and the DNA-protein complexes were separated on a $6 \%$ native polyacrylamide gel. GST protein alone was used as the negative control.+or ++ indicate increasing amounts of unlabeled or mutant probe used for competition and testing of binding specificity. Arrows indicate the position of the shifted bands.

a

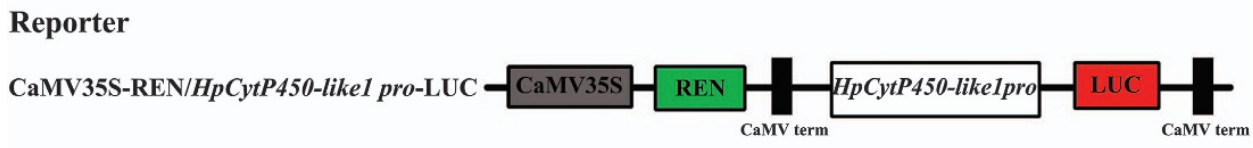

Effector

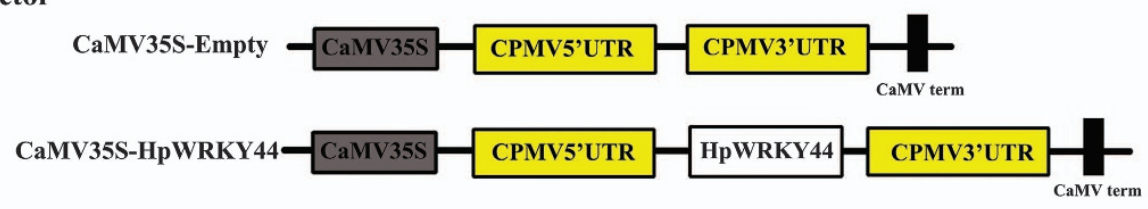

b

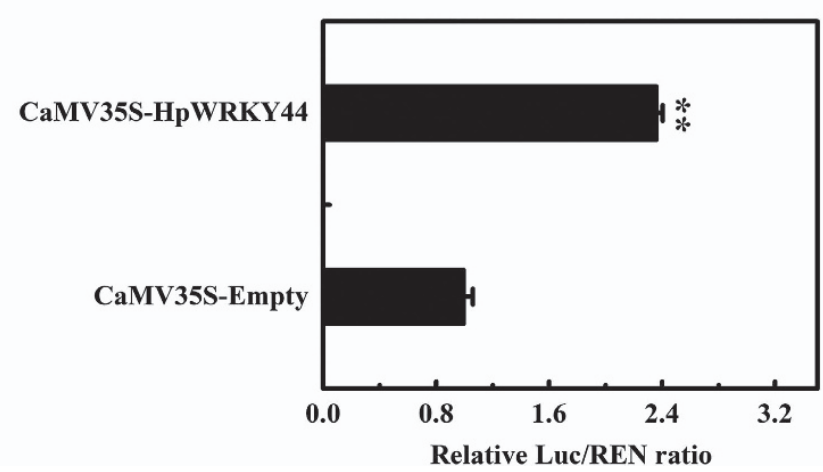

Figure 7. HpWRKY44 directly activates the expression of HpCytP450-like1. (a) Diagrammatic illustrations of the reporter and effector constructs used in the transient dual-luciferase reporter assay in tobacco leaves. LUC, firefly luciferase; REN, renilla luciferase; CPMV, Cowpea mosaic virus. (b) HpWRKY44 trans-activates the HpCytP450-like1 promoter. The activation was assessed by the ratio of LUC to REN. The ratio of LUC/REN of the empty vector plus promoter was used as a calibrator and set at 1. Each presented value represents the mean \pm s.e.m. six biological replicates $(n=6) .{ }^{* *}$ Indicates statistically significant differences at $P<0.01$, as determined by Student's $t$-test.

biosynthesis in pitayas; which are economically important and nutritious fruit.

\section{CONFLICT OF INTEREST}

The authors declare no conflict of interest.

\section{ACKNOWLEDGEMENTS}

We thank Professor George P. Lomonossoff (Department of Biological Chemistry, John Innes Centre, Norwich Research Park) for providing the $\mathrm{PEAQ}$ vectors, and $\mathrm{Dr}$ Prakash Lakshmanan (Sugar Research Australia) for improving the language of the manuscript. This work was supported by the Science and Technology Planning Project of Guangdong Province (2014B020202010), Science and Technology Program of Guangzhou (201704020003 and 2014Y2-00164), YangFan Innovative and Entepreneurial Research Team Project (2014YT02H013) and the earmarked fund for Guangdong Modern Agro-industry Technology Research System (2016LM112).

\section{REFERENCES}

1 Gandía-Herrero F, García-Carmona F. Biosynthesis of betalains: yellow and violet plant pigments. Trends Plant Sci 2013; 18: 334-343.

2 Khan MI, Giridhar P. Plant betalains: chemistry and biochemistry. Phytochem 2015; 117: $267-295$.

3 Gandía-Herrero F, Escribano J, García-Carmona F. Biological activities of plant pigments betalains. Crit Rev Food Sci Nutr 2016; 56: 937-945.
4 Gandía-Herrero F, Escribano J, García-Carmona F. Purification and antiradical properties of the structural unit of betalains. J Nat Prod 2012; 75: 1030-1036.

5 Stafford HA. Anthocyanins and betalains: evolution of the mutually exclusive pathways. Plant Sci 1994; 101: 91-98.

6 Clifford T, Howatson G, West DJ, Stevenson EJ. The potential benefits of red beetroot supplementation in health and disease. Nutrients 2015; 7: 2801-2822.

7 Azeredo HMC. Betalains: properties, sources, applications, and stability-a review. Int J Food Sci Technol 2009; 44: 2365-2376.

$8 \mathrm{Han}$ XH, Gao ZJ, Xiao XG. Enzymes and genes involved in the betalain biosynthesis in higher plants. Afr J Biotechnol 2009; 8: 6735-6744.

9 Hatlestad GJ, Sunnadeniya RM, Akhavan NA et al. The beet R locus encodes a new cytochrome P450 required for red betalain production. Nat Genet 2012; 44: 816-820.

10 Hua QZ, Chen $\mathrm{CJ}$, Chen Z et al. Transcriptomic analysis reveals key genes related to betalain biosynthesis in pulp coloration of Hylocereus polyrhizus. Front Plant Sci 2016; 6: 1179.

11 DeLoache WC, Russ ZN, Narcross L, Gonzales AM, Martin VJ, Dueber JE. An enzyme-coupled biosensor enables (S)-reticuline production in yeast from glucose. Nat Chem Biol 2015; 11: 465-471.

12 Polturak G, Breitel D, Grossman N et al. Elucidation of the first committed step in betalain biosynthesis enables the heterologous engineering of betalain pigments in plants. New Phytol 2016; 210: 269-283.

13 Sunnadeniya R, Bean A, Brown M et al. Tyrosine hydroxylation in betalain pigment biosynthesis is performed by cytochrome P450 enzymes in beets (Beta vulgaris). PLOS ONE 2016; 11: e0149417.

14 Hatlestad GJ, Akhavan NA, Sunnadeniya RM et al. The beet $Y$ locus encodes an anthocyanin MYB-like protein that activates the betalain red pigment pathway. Nat Genet 2015; 47: 92-96. 
15 Ishiguro S, Nakamura K. Characterization of a CDNA encoding a novel DNAbinding protein, SPF1, that recognizes SP8 sequences in the $5^{\prime}$ upstream regions of genes coding for sporamin and beta-amylase from sweet potato. Mol Gen Genet 1994; 244: 563-571.

16 Rushton PJ, Somssich IE, Ringler P, Shen QJ. WRKY transcription factors. Trends Plant Sci 2010; 15: 247-258.

17 Rushton DL, Tripathi P, Rabara RC et al. WRKY transcription factors: key components in abscisic acid signalling. Plant Biotechnol J 2012; 10: 2-11.

18 Chen L, Song Y, Li S, Zhang L, Zou C, Yu D. The role of WRKY transcription factors in plant abiotic stresses. Biochim Biophys Acta 2012; 1819: 120-128.

19 Phukan UJ, Jeena GS, Shukla RK. WRKY transcription factors: molecular regulation and stress responses in plants. Front Plant Sci 2016; 7: 760.

20 Jiang J, Ma S, Ye N, Jiang M, Cao J, Zhang J. WRKY transcription factors in plant responses to stresses. J Integr Plant Biol 2017; 59: 86-101.

21 Eulgem T, Rushton PJ, Robatzek S, Somssich IE. The WRKY superfamily of plant transcription factors. Trends Plant Sci 2000; 5: 199-206.

22 Song $Y, A i C R$, Jing SJ, Yu DQ. Research progress on functional analysis of rice WRKY genes. Rice Sci 2010; 17: 60-72.

23 Zhou X, Jiang Y, Yu D. WRKY22 transcription factor mediates dark-induced leaf senescence in Arabidopsis. Mol Cells 2011; 31: 303-313.

24 Bakshi M, Oelmüller R. WRKY transcription factors. Plant Signal Behav 2014; 9: e27700.

25 Kato N, Dubouzet E, Kokabu Y et al. Identification of a WRKY protein as a transcriptional regulator of benzylisoquinoline alkaloid biosynthesis in Coptis japonica. Plant Cell Physiol 2007; 48: 8-18.

26 Ma D, Pu G, Lei $C$ et al. Isolation and characterization of AaWRKY1, an Artemisia annua transcription factor that regulates the amorpha-4,11-diene synthase gene, a key gene of artemisinin biosynthesis. Plant Cell Physiol 2009; 50: 2146-2161.

27 Suttipanta N, Pattanaik S, Kulshrestha M, Patra B, Singh SK, Yuan L. The transcription factor CrWRKY1 positively regulates the terpenoid indole alkaloid biosynthesis in Catharanthus roseus. Plant Physiol 2011; 157: 2081-2093.

28 Schluttenhofer C, Yuan L. Regulation of specialized metabolism by WRKY transcription factors. Plant Physiol 2015; 167: 295-306.

29 García-Cruz L, Valle-Guadarrama S, Salinas-Moreno Y, Joaquín-Cruz E. Physical, chemical, and antioxidant activity characterization of pitaya (Stenocereus pruinosus) fruits. Plant Foods Hum Nutr 2013; 68: 403-410.

30 Suh $\mathrm{DH}$, Lee $\mathrm{S}$, Heo do $\mathrm{Y}$ et al. Metabolite profiling of red and white pitayas (Hylocereus polyrhizus and Hylocereus undatus) for comparing betalain biosynthesis and antioxidant activity. J Agric Food Chem 2014; 62: 8764-8771.

31 Wybraniec S, Stalica P, Jerz G et al. Separation of polar betalain pigments from cacti fruits of Hylocereus polyrhizus by ion-pair high-speed countercurrent chromatography. J Chromatogr A 2009; 1216: 6890-6899.

32 Sainsbury F, Thuenemann EC, Lomonossoff GP. pEAQ: versatile expression vectors for easy and quick transient expression of heterologous proteins in plants. Plant Biotechnol J 2009; 7: 682-693.
33 Fan ZQ, Kuang JF, Fu CC et al. The banana transcriptional repressor MaDEAR1 negatively regulates cell wall-modifying genes involved in fruit ripening. Front Plant Sci 2016; 7: 1021.

34 Healey A, Furtado A, Cooper T, Henry RJ. Protocol: a simple method for extracting next-generation sequencing quality genomic DNA from recalcitrant plant species. Plant Methods 2014; 10: 21.

35 Fu CC, Han YC, Qi XY et al. Papaya CpERF9 acts as a transcriptional repressor of cell-wall-modifying genes CpPME1/2 and CpPG5 involved in fruit ripening. Plant Cell Rep 2016; 35: 2341-2352.

36 Hellens RP, Allan AC, Friel EN et al. Transient expression vectors for functional genomics, quantification of promoter activity and RNA silencing in plants. Plant Methods 2005; 1: 13.

37 Johnson CS, Kolevski B, Smyth DR. TRANSPARENT TESTA GLABRA2, a trichome and seed coat development gene of Arabidopsis, encodes a WRKY transcription factor. Plant Cell 2002; 14: 1359-1375.

38 Mao G, Meng X, Liu Y, Zheng Z, Chen Z, Zhang S. Phosphorylation of a WRKY transcription factor by two pathogen-responsive MAPKs drives phytoalexin biosynthesis in Arabidopsis. Plant Cell 2011; 23: 1639-1653.

39 Gong X, Zhang J, Hu J et al. FcWRKY70, a WRKY protein of Fortunella crassifolia, functions in drought tolerance and modulates putrescine synthesis by regulating arginine decarboxylase gene. Plant Cell Environ 2015; 38: 2248-2262.

40 Ye YJ, Xiao YY, Han YC et al. Banana fruit VQ motif-containing protein5 represses cold-responsive transcription factor MaWRKY26 involved in the regulation of JA biosynthetic genes. Sci Rep 2016; 6: 23632

41 Ulker B, Somssich IE. WRKY transcription factors: from DNA binding towards biological function. Curr Opin Plant Biol 2004; 7: 491-498.

42 Chen M, Yan T, Shen Q et al. GLANDULAR TRICHOME-SPECIFIC WRKY 1 promotes artemisinin biosynthesis in Artemisia annua. New Phytol 2017; 214: 304-316.

43 Chi Y, Yang Y, Zhou Y et al. Protein-protein interactions in the regulation of WRKY transcription factors. Mol Plant 2013; 6: 287-300.

44 Shan W, Chen JY, Kuang JF, Lu WJ. Banana fruit NAC transcription factor MaNAC5 cooperates with MaWRKYs to enhance the expression of pathogenesis-related genes against Colletotrichum musae. Mol Plant Pathol 2016; 17: 330-338.

\section{c) (i)}

This work is licensed under a Creative Commons Attribution 4.0 International License. The images or other third party material in this article are included in the article's Creative Commons license, unless indicated otherwise in the credit line; if the material is not included under the Creative Commons license, users will need to obtain permission from the license holder to reproduce the material. To view a copy of this license, visit http://creativecommons.org/licenses/ by/4.0/

(c) The Author(s) 2017

Supplementary Information for this article can be found on the Horticulture Research website (http://www.nature.com/hortres) 